\title{
Avaliação das estruturas de cobertura em madeira de um galpão de estoque de produtos químicos
}

\author{
Evaluation of wood roof structures of industrial shed for \\ chemicals stock
}

\section{Jairo Ribas Andrade Junior Diego Henrique de Almeida Tiago Hendrigo de Almeida André Luis Christoforo Guilherme Corrêa Stamato Francisco Antonio Rocco Lahr}

\section{Resumo}

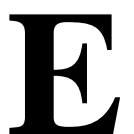

ste trabalho tem com objetivo avaliar a integridade das estruturas que fazem parte da cobertura de um galpão de 70 anos, destinado ao estoque de produtos químicos, localizada no Estado de São Paulo (Brasil), e apontar possíveis soluções para os problemas identificados. Buscou-se estimar a influência dos resíduos dos produtos químicos depositados nas superfícies dos elementos estruturais e identificar a espécie de madeira utilizada no projeto, por meio de ensaios físicos (densidade aparente) e mecânicos (compressão e cisalhamento paralelo às fibras. Os problemas identificados foram: (a) umidade devido a telhas quebradas; (b) deterioração superficial da madeira em função de reação química entre os produtos armazenados e a estrutura; (c) cavilhas de madeira com instalação incompleta; (d) falta de mãos-francesas em algumas terças; (e) travamento inadequado das treliças; e (f) deterioração de pregos de fixação por oxidação. Pelas observações realizadas, as soluções indicadas são de fácil execução, pois as estruturas não foram severamente prejudicadas. Com relação à espécie de madeira, os resultados das propriedades mecânicas obtidas associadas à densidade aparente apontaram para a espécie Peroba-Rosa (Aspidosperma polyneuron). A análise estatística de resultados apontou para a não influência do produto químico no desempenho da estrutura do telhado.

Diego Henrique de Almeida Pontifícia Universidade Católica de Minas Gerais

Poços de Caldas - MG - Brasil

Palavras-chave:Madeira. Estruturas de cobertura. Galpõesindustriais.

Deterioração. Vistoria.

\section{Abstract}

Tiago Hendrigo de Almeida Universidade de São Paulo São Carlos - SP - Brasil

André Luis Christoforo Universidade Federal de São Carlos São Carlos - SP - Brasil

Guilherme Corrêa Stamato Stamade - Projeto e Consultoria em Madeira Ltda. São Carlos - SP - Brasil

Francisco Antonio Rocco Lahr Universidade de São Paulo São Carlos - SP - Brasil

Recebido em 16/11/13 Aceito em 11/08/14
This research work aims to assess the structural integrity of 70-year old wood roof components of a industrial shed for chemicals stock, located in the State of São Paulo (Brazil), as well as to point out possible solutions for the existing problems. This study estimated the influence of chemicals deposited on the surface of the structural elements, and identified the wood species used in the design, by performing physical (density) and mechanical tests (compression strength, and shear strength in the parallel direction to the grain). The problems identified were: (a) moisture due to broken tiles; (b) deterioration of the wood surface due to the chemical reaction between the stored products and structure; (c) wood-dowels with incomplete installation; (d) lack of supporting elements in some of the purlins; (e) irregular perpendicular bracing of trusses; and $(f)$ deterioration of the fastening nails by oxidation. Based on the direct observation of the problems, most solutions seem to be relatively easy to perform, since the structures were not severely damaged. Regarding the wood species, the results of mechanical properties associated with physical characteristics indicated that Peroba-Rosa (Aspidospermapolyneuron) wood species was used. The statistical analysis indicated that the influence of the chemical products in the performance of the roof structure was not considered to be significant.

Keywords: Wood. Roof structures. Industrial sheds. Deterioration. Inspection.

ANDRADE JUNIOR, J. R.; ALMEIDA, D. H. de; ALMEIDA, T. H. de; CHRISTOFORO, A. L.; STAMATO, G. C.; LAHR, R. A. 75 R.Avaliação das estruturas de cobertura em madeira de um galpão de estoque de produtos químicos. Ambiente Construído, Porto Alegre, v. 14, n. 3, p. 75-85, jul./set. 2014.

ISSN 1678-8621 Associação Nacional de Tecnologia do Ambiente Construído. 


\section{Introdução}

A madeira é um dos principais materiais utilizados na construção civil por causa dafacilidade e versatilidade de seu emprego. Podem ser utilizadas madeiras provenientes de florestas plantadas, principalmente dos gêneros Pinus, Eucalyptus, Tectona e Corymbia,além daquelas que têm sido objeto de estudo acerca de suas propriedades mecânicas visando à utilização na construção civil. Além disso, o forte apelo socioambiental fornece à madeira vantagens em relação a outros materiais empregados na construção civil (CALIL JUNIOR; LAHR; DIAS, 2003).

$\mathrm{O}$ uso da madeira como material estrutural requer o conhecimento de suas propriedades física e mecânicas, pois são estas que interferem no projeto de estruturas de madeira (ALMEIDA et al., 2011; FERROet al., 2013; ICIMOTO et al., 2013a).

Por ser a madeira um material higroscópico, o teor de umidade também é um fator preponderante nas construções, e variação da umidade abaixo do ponto de saturação das fibras impõe variação nas propriedades mecânicas (GALVÃO; JANKOWSKY, 1985). As propriedades de resistência e de rigidez de peças estruturais de madeira diminuem com o aumento do teor de umidade até o ponto de saturação das fibras (PSF), que é em torno de 25\% (LOGSDON; CALIL JUNIOR, 2002; SCALIANTE et al., 2011).

É importante destacar que a avaliação do desempenho dos elementos estruturais deve ser conduzida com base na norma da Associação Brasileira de Normas Técnicas (ABNT) NBR 7190 (ABNT, 1997) - Projeto de Estruturas de Madeira, de modo a garantir os níveis de segurança estabelecidos (OLIVEIRA, 2001; CALIL JUNIOR; MOLINA, 2010; ALMEIDA, 2011; ALMEIDA et al., 2013a; 2013b).

Estruturas construídas com madeira não tratada contra a demanda biológica, com o decorrer dos anos, poderão apresentar patologia devida ao ataque de cupins, brocas e fungos. Tais ataques podem ser evitados com o uso de tratamentos preservantes, sendo os mais usuais no Brasil aqueles com soluções salinas de CCA (sais de cobre, cromo e arsênio) e CCB (sais de cobre, cromo e boro), conforme apontado por Bertolini et al. (2013) e Icimoto et al. (2013b).

Nesse contexto, o presente trabalho teve como objetivo avaliar as condições das estruturas de cobertura de galpão (construído há setenta anos) para estocagem de produtos químicos, por intermédio de vistoria e ensaios de laboratório, bem como identificar a espécie de madeira utilizada na referida construção.

\section{Material e métodos}

As vistorias nas estruturas de cobertura do galpão de armazenamento de produtos químicos ocorreram no final do primeiro semestre de 2013. Para determinar a situação das estruturas em relação às possíveis patologias, foram realizadas análises visuais in loco em todos os componentes estruturais em madeira e nas ligações das estruturas (efetuadas com elementos metálicos). $\mathrm{Na}$ vistoria, foram avaliadas as condições estruturais gerais, os detalhes de fixação e as condições de deterioração em uso da estrutura, apontando soluções para os problemas encontrados.

Como a empresa não possui os projetos de construção originais dessas estruturas de cobertura,para investigar a influência do ataque proveniente da deposição de material químico sobre a superfície da madeira e a devida identificação da espécie utilizada no projeto foram utilizadas algumas peças de madeira armazenadas no galpão (6 caibros de $5 \times 6 \times 130 \mathrm{~cm})$, estando parcialmente atingidas pela ação dos produtos químicos. Das peças de madeira foram extraídos corpos de prova para análise de algumas propriedades mecânicas e da densidade aparente $\left(\rho_{a p}\right)$. Entre as propriedades mecânicas avaliadas estão: resistência à compressão paralela às fibras $\left(f_{c 0}\right)$ e resistência ao cisalhamento paralelo às fibras $\left(f_{v 0}\right)$. Os ensaios para obtenção da densidade aparente e das propriedades mecânicas foram realizados seguindo as premissas e métodos de cálculo da Norma Brasileira NBR 7190 (ABNT, 1997), executados nas dependências do Laboratório de Madeiras e Estruturas de Madeira (LaMEM), do Departamento de Engenharia de Estruturas, da Escola de Engenharia de São Carlos, da Universidade de São Paulo (EESC/USP). A identificação da espécie de madeira também se deu com o uso de fichas catalogadas de espécies de madeira do Instituto de Pesquisas Tecnológicas (INSTITUTO..., 2013). Os corpos de prova para os ensaios de resistência à compressão paralela às fibras e de resistência ao cisalhamento paralelo às fibras são ilustrados nas Figuras 1 e 2 respectivamente.

76 Andrade Junior, J. R.; Almeida, D. H. de; Almeida, T. H. de; Christoforo, A. L.; Stamato, G. C.; Lahr, R. A. R. 
Figura 1 - Corpo de prova para o ensaio de compressão paralela às fibras

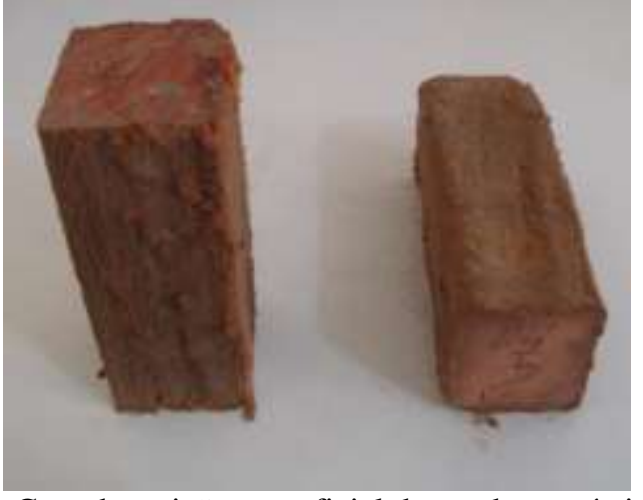

(a) Com deposição superficial do produto químico

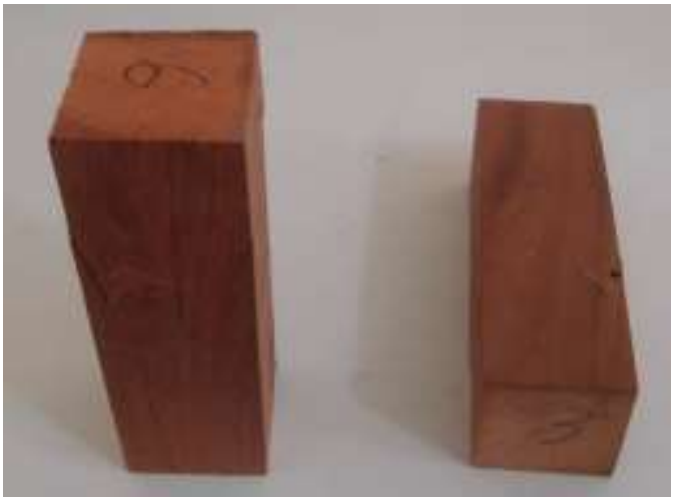

(b) Posteriormente aparelhado

Figura 2 - Corpo de prova para ensaio de cisalhamento paralelo às fibras

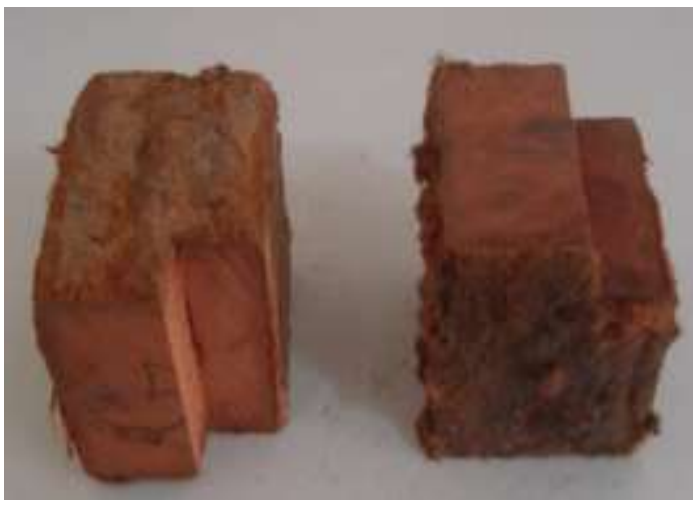

(a) Com deposição superficial do produto químico

Os detalhes das características construtivas da estrutura avaliada são:

(a) treliças:estruturas principais compostasdos banzos superiores e inferiores, montantes e diagonais, com as ações verticais e horizontais transferidas para a fundação por meio dos pilares;

(b) banzo superior: seção transversal composta de três peças paralelas de $6 \times 16 \mathrm{~cm}$ com reforço superior de 2 peçasdeitadas de $3 \times 16 \mathrm{~cm}$.

(c) banzo inferior: seção transversal composta de três peças paralelas de $6 \times 16 \mathrm{~cm}$.

(d) diagonais: as quatro primeiras diagonais a partir da base e as três últimas diagonais são reforçadas. Comprimento de $3 \mathrm{~m}$ e seção transversal variando entre 3 peças paralelas de $6 \times 12 \mathrm{~cm}$ em alguns pontos e em outros com 2 peças paralelas $6 \times 12 \mathrm{~cm}$, alguns desses com reforço de $3 \times 16 \mathrm{~cm}$ em casos específicos;

(e) montantes: comprimento de 1,90m e seção transversal composta de duas peças paralelas $6 \times 12 \mathrm{~cm}$, com exceção nos pontos de emenda dos banzos;

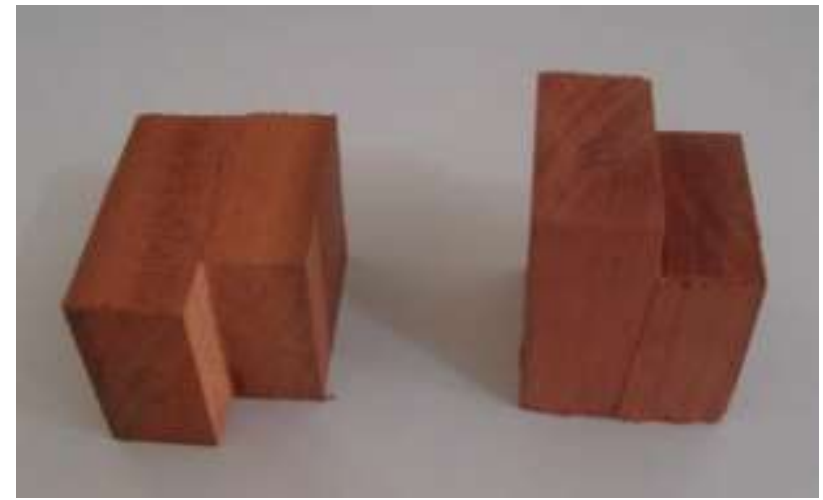

(b) Posteriormente aparelhado

(f) ligações: todas as ligações entre os elementos componentes da treliça foram executadas com cavilhas de madeira com diâmetro de 3 e 1/4", com uma cavilha por ligação, com exceções para emenda do banzo superior ( 9 cavilhas) e emenda do banzo inferior (13 cavilhas). Os nós de treliças contam também com entalhes que transferem os esforços entre as diagonais e montantes;

(g) mãos-francesas: as treliças são travadas entre o banzo inferior e as terças por meio de mãosfrancesas com seção transversal de $6 \times 6 \mathrm{~cm}$, para garantir a estabilidade das treliças em seus planos;

(h) travamento global no plano do telhado: travamento contínuo a partir do beiral até a cumeeira, conectando toda a estrutura aos pilares de sustentação.Seção transversal de perfil em " $T$ " formado por duas peças $(6 \times 8+3 \times 11) \mathrm{cm}$, apresentando comprimento de $6,00 \mathrm{~m}$ entre cada treliça, contínuo do beiral à cumeeira;

(i) travamento de extremidades no plano do telhado: travamentos no plano do telhado dispostos em " $\mathrm{X}$ ” em todos os vãos de terças e apenas as treliças das extremidades, com a função de evitar o colapso da estrutura devido à concentração de 
esforços horizontais no último pórtico. Seção transversal em perfil de "T" formado por duas peças $(6 \times 8+3 \times 11) \mathrm{cm}, \quad$ com comprimento variando em função de sua localização na estrutura; e

(j) linhal no plano do telhado: travamento das terças do frechal à cumeeira, para evitar a flecha das terças na menor inércia devido à alta inclinação da cobertura. Seção transversal de $6 \times 6 \mathrm{~cm}$ e comprimento de $1,65 \mathrm{~m}$.

A investigação da influência dos produtos químicos nas propriedades física e mecânicas foi feita por meio de análise de variância (ANOVA), com o auxílio do software Minitab ${ }^{\circledR}$ versão 14 , considerado o nível de significância $(\alpha)$ de 5\%. A equivalência entre os valores médios das propriedades foi adotada como hipótese nula $\left(H_{0}\right)$, e a não equivalência, como hipótese alternativa $\left(H_{l}\right)$. Caso os p-valoressejam inferiores ao nível de significância, deve-se rejeitar $H_{0}$, aceitando-a em caso contrário.

Para validação da ANOVA, foram avaliadas a normalidade (teste de Anderson-Darling) nas distribuiçõesdas variáveis-resposta e a homogeneidade entre variâncias dos dois tratamentos (testes $\mathrm{F}$ e de Levene). Ambos os testes foram considerados ao nível de $5 \%$ de significância. O teste de Anderson-Darling teve como hipótese nula a normalidade nas distribuições, e a não normalidade como hipótese alternativa. Caso os p-valoressejam superiores ao nível de significância, deve-se aceitar $H_{0}$, refutando-a em caso contrário. Os testes $\mathrm{F}$ e Levene tiveram a equivalência das variâncias entre os tratamentos como hipótese nula, e a não equivalência como hipótese alternativa. Parapvalores superiores ao nível de significância, devese aceitar $H_{0}$, refutando-a em caso contrário.

\section{Resultados e discussão}

\section{Inspeção visual}

$\mathrm{Na}$ inspeção visual foi constatado que, globalmente, a estrutura se encontra estável, com ressalva para alguns pontos descritos a seguir. As ações sugeridas devem ser realizadas para a melhoria das instalações:

PROBLEMA1: pontos de umidade na estrutura de cobertura, devido a telhas quebradas ou desencaixadas, ilustrados na Figura 3.

$A C ̧ \tilde{A} O$ 1: deve-se realizar manutenção periódica na cobertura da estrutura, para substituição de telhas com trincas ou quebradas, evitando-se pontos de entrada de umidade que propiciem deterioração mais acelerada da estrutura.

PROBLEMA 2: deterioração superficial da madeira em função de reação química entre os produtos armazenados (fertilizantes com várias proporções de ingredientes químicos) e a estrutura (Figura 4). Uma análise detalhada do efeito dessa deterioração superficial foi realizada a partir de ensaios de resistência mecânica, descritos em detalhes mais à frente.

$A C ̧ \tilde{A} O$ 2:a deterioração é decorrente de partículas em suspensão de alguns dos elementos químicos componentes dos fertilizantes que se precipitam e ficam acumulados sobre a madeira,sendo recomendada a limpeza, com periodicidade de 1 ano, das estruturas, para remoção das camadas de sólidos acumulados.

PROBLEMA 3: Cavilhas com instalação incompleta, estando algumas ligações prejudicadas pela fixação parcial das cavilhas que não abrangem todas as peças do conjunto, dificultando a transferência das forças entre os elementos da treliça. Pode ser verificado que a grande maioria das cavilhas está intacta. Conclui-se que as encontradas com falhas são decorrentes da instalação mal realizada, e não de deterioração com o tempo(Figura5).

Figura 3 - Pontos de umidade na estrutura de cobertura

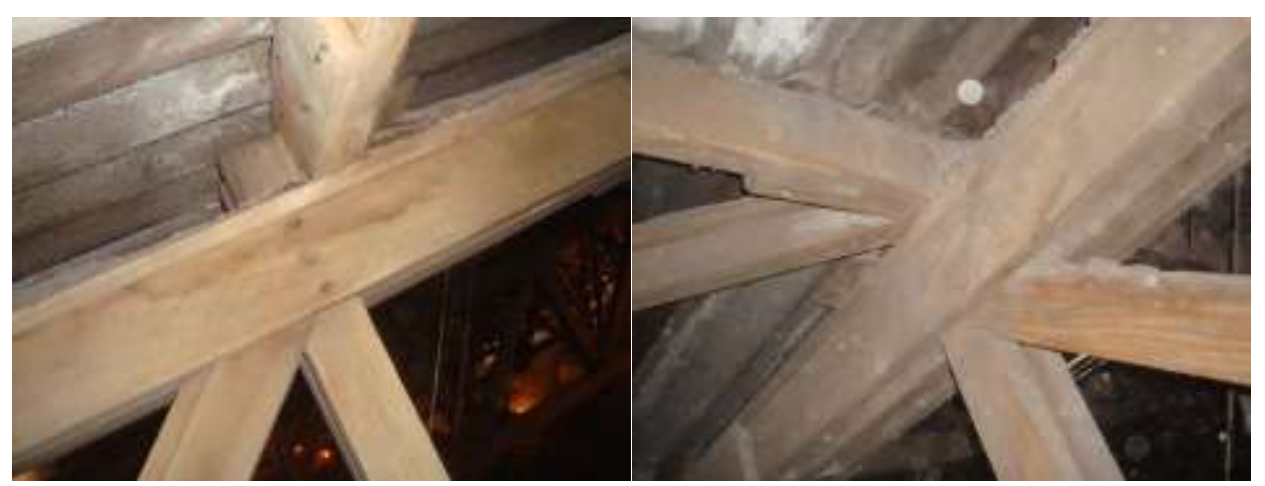

78 Andrade Junior, J. R.; Almeida, D. H. de; Almeida, T. H. de; Christoforo, A. L.; Stamato, G. C.; Lahr, R. A. R. 
Figura 4 - Acúmulo de material químico na superfície dos elementos estruturais

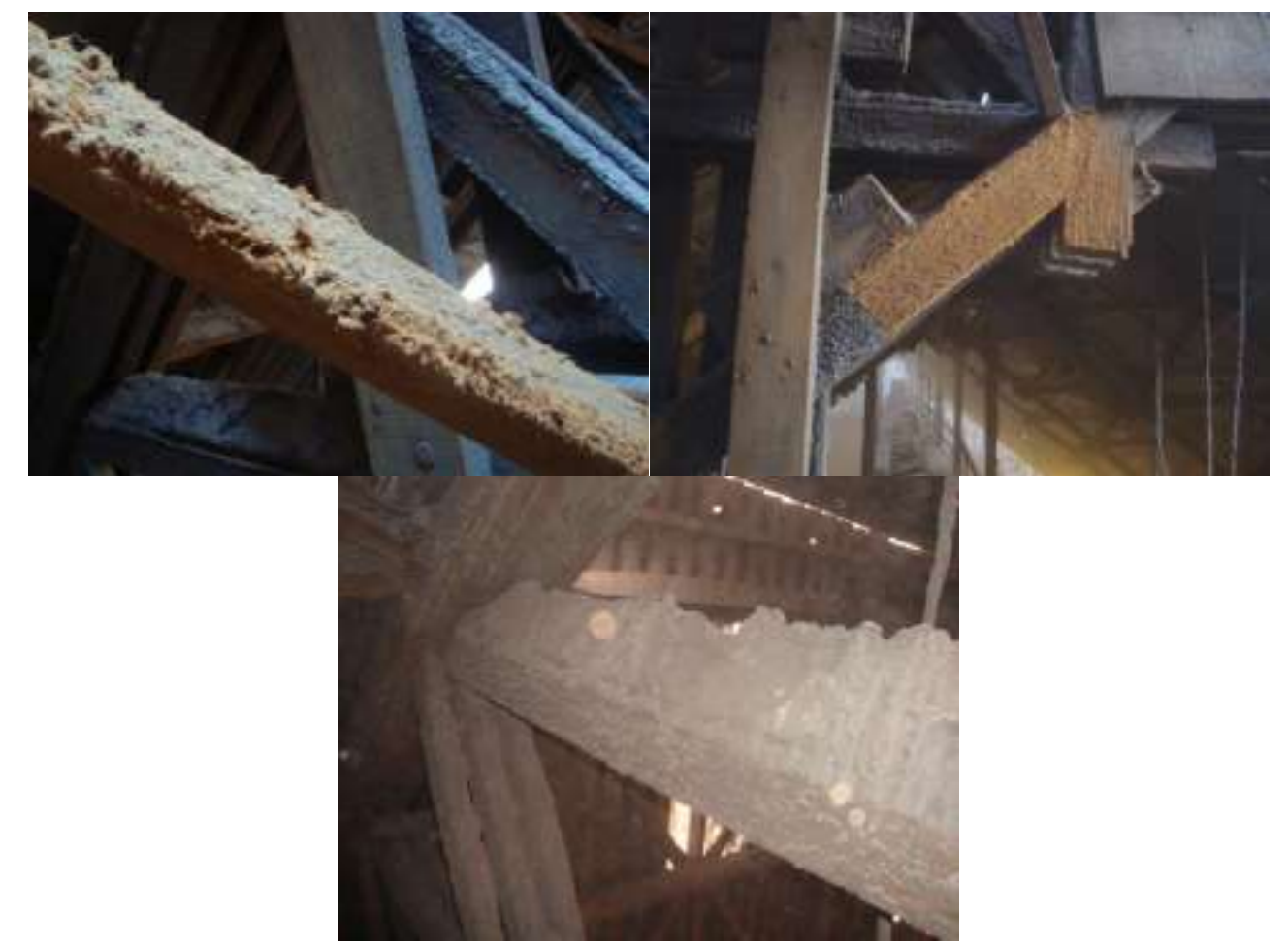

Figura 5 - Cavilhas degradadas

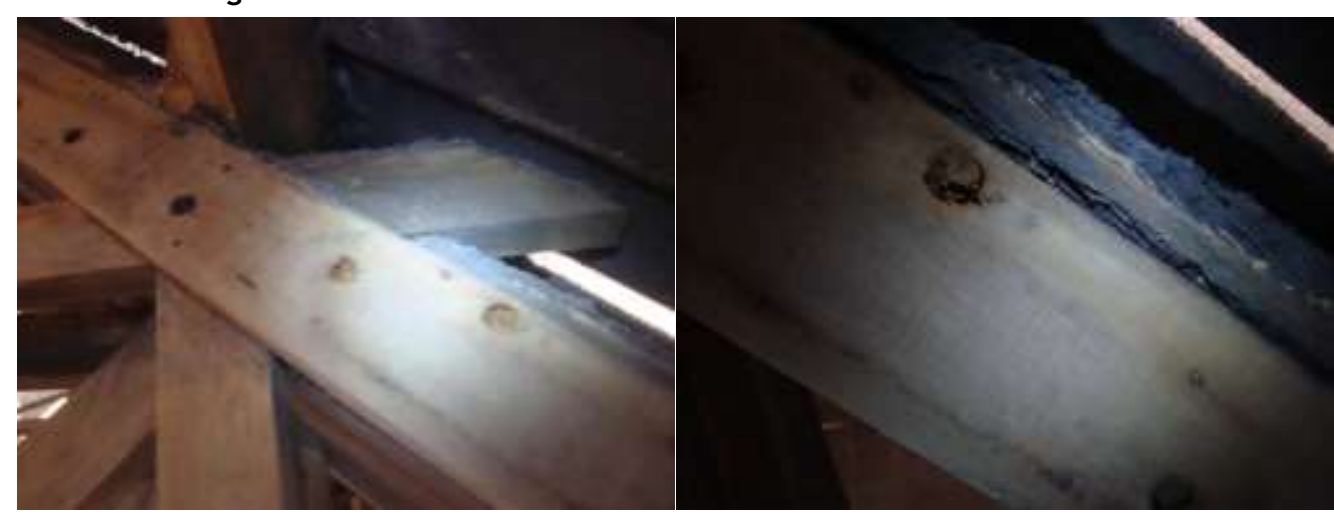

$A C ̧ \tilde{A} O$ 3: É necessária a substituição dessas cavilhas, com inserção de cavilhas novas, da mesma dimensão e confeccionadas com madeira de classe de resistência C60 (Cumaru, Jatobá ou Maçaranduba) da norma Brasileira NBR 7190 (ABNT, 1997). A substituição se faz necessária para a recomposição da resistência dessas ligações para as solicitações de projeto. Caso haja impossibilidade de utilização de novas cavilhas de madeira, pode-se utilizar conectores metálicos, como parafusos com porcas e arruelas ou chapas metálicas.

PROBLEMA 4: Falta de mãos-francesas nas terças mais próximas à base, extraídas, possivelmente devido a impactos de maquinários de movimentação de material. Em pontos mais elevados, algumas mãos-francesas estão ausentes devido à deterioração dos pregos metálicos de fixação, que se soltaram, o que representa perigo aos que transitam sob a estrutura (Figura 6).

$A C ̧ \tilde{A} O$ 4: É necessária a substituição das mãosfrancesas soltas e a reposição das faltantes, antes de executar qualquer outra intervenção na estrutura, pois essas peças são responsáveis pelo travamento na direção horizontal. Para recolocação de mãos-francesas sugere-se utilizar pregos de inox ardox ou anelados.

PROBLEMA 5:As treliças que tiveram os travamentos verticais suprimidos tendem a apresentar desvio lateral, que instabilizam a 
estrutura. Isso já está ocorrendo em algumas das treliças analisadas (Figura 7).

$A C ̧ \tilde{A} O$ 5:É necessária a intervenção nesses apoios das treliças, substituindo todas as peças danificadas até o $5^{\circ}$ montante, pois as deformações já são excessivas, e as intervenções anteriores já alteraram o comportamento original dessas peças. Em todos os apoios das treliças deve ser realizado o travamento adequado, para garantir a estabilidade lateral.

Figura 6 -(a) Travamento no plano do telhado solto por degradação do prego; (b)mão-francesa quebrada por impacto; (c) mão-francesa solta por impacto ou por degradação da ligação; e (d)base do montante quebrada para instalação da divisória do box

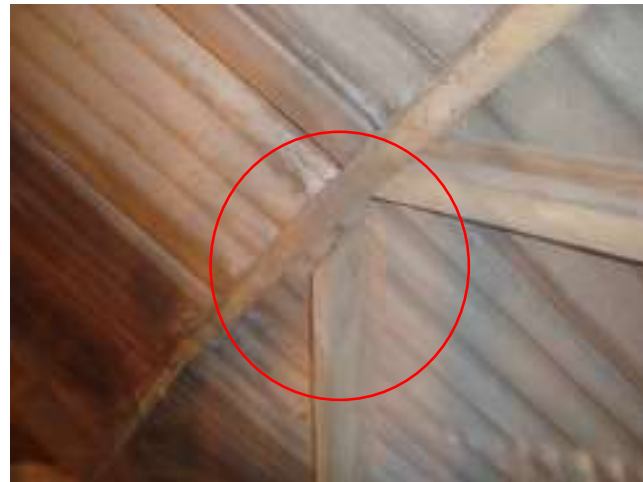

(a)

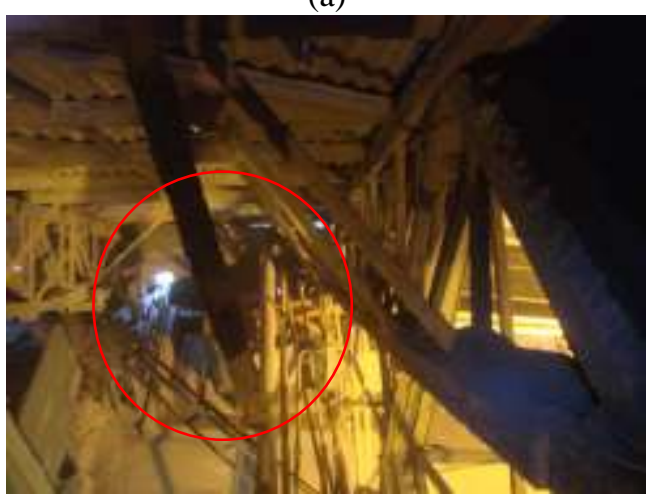

(c)

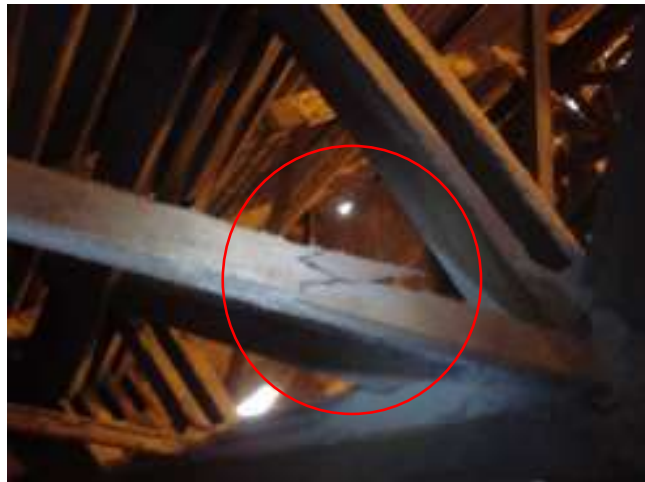

(b)

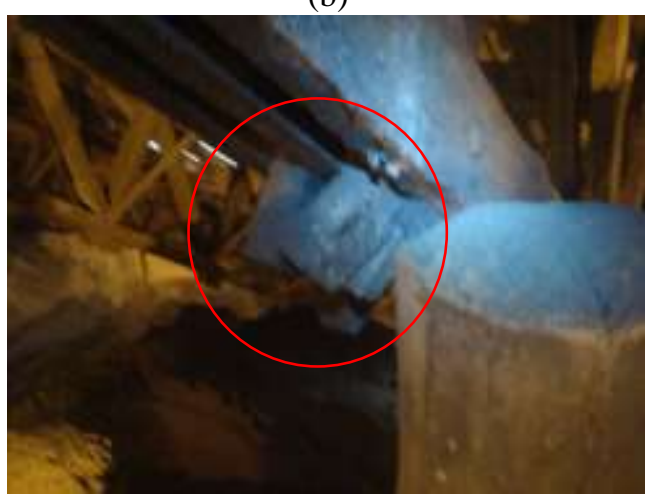

(d)

Figura 7 - Flambagem de treliças por deficiência no travamento

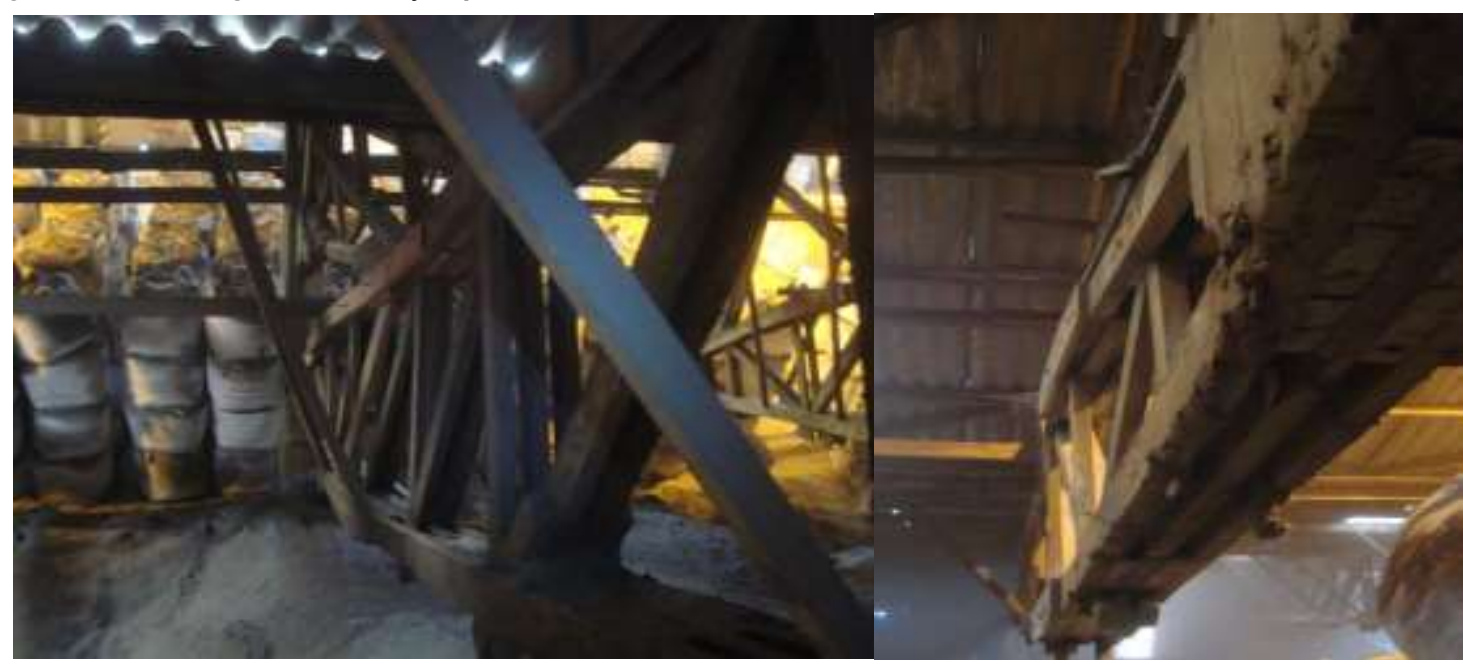

80 Andrade Junior, J. R.; Almeida, D. H. de; Almeida, T. H. de; Christoforo, A. L.; Stamato, G. C.; Lahr, R. A. R. 
PROBLEMA 6: Diversos pregos de fixação de mãos-francesas apresentam elevado grau de deterioração por oxidação, devida à reação química com os materiais armazenados (Figura 8).

$A C ̧ \tilde{A} O$ 6:Imprescidível verificar periodicamente as mãos-francesas e, conforme for necessária a substituição, utilizar pregos de inox nos novos travamentos.

\section{Identificação da madeira e avaliação das diferenças nas resistências mecânicas}

Utilizando as fichas catalogadas de espécies de madeira disponíveis no site do Instituto de Pesquisas Tecnológicas (INSTITUTO..., 2013), ficou mais claro de qual madeira se tratava, sendo as características macroscópicas da utilizada na cobertura do galpão compatíveis com a PerobaRosa (Aspidosperma polyneuron), assim como ilustrado na Figura 9. A Figura 9(c) apresenta uma fotomacrografia aumentada em 10x.

A Tabela 1 apresenta os resultados das propriedades mecânicas e da densidade aparente obtidos para cada um dos corpos de prova (CP), os valores médios amostrais (x), os desvios padrão $(\mathrm{Sd})$ e os coeficientes de variação $(\mathrm{Cv})$ para as condições de madeira encoberta (Det) e não encoberta pelo produto químico (Non Det.).

As Figuras 10 e 11 ilustram os resultados dos testes de normalidade e de homogeneidade entre variâncias respectivamente. Pelo fato de os pvalores encontrados serem ambos superiores a 0,05 , constata-se que as distribuições podem ser consideradas normais e que as variâncias entre os tratamentos são homogêneas, validando o modelo da ANOVA.

Figura 8 - Prego deteriorado na ligação da mão-francesa com grande trinca

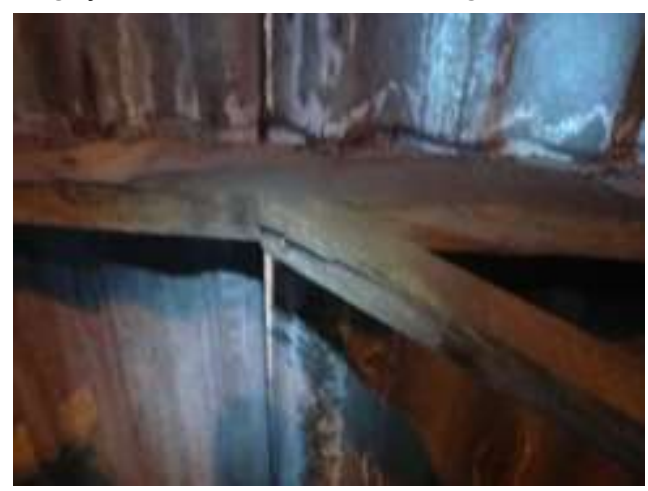

Figura 9 -Peroba-Rosa

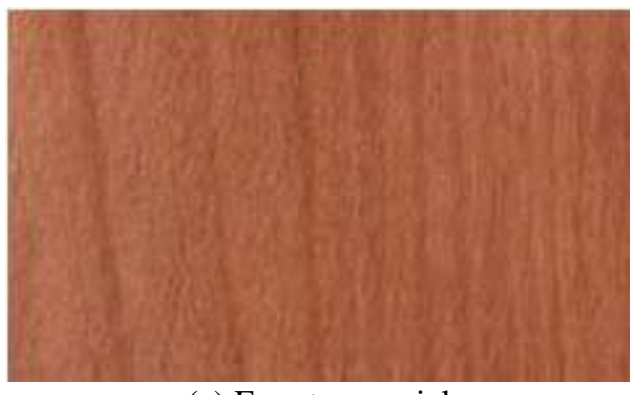

(a) Face tangencial

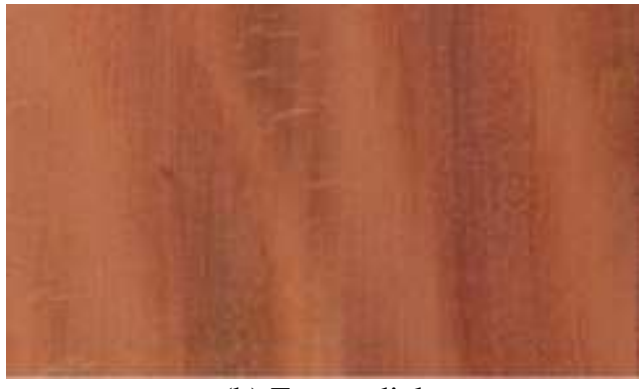

(b) Face radial

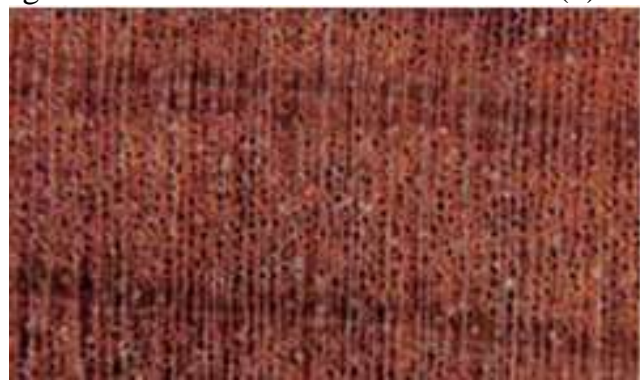

(c) Fotomacrografia com apliação de 10x

Fonte: Instituto de Pesquisas Tecnológicas (2013). 
Tabela 1 - Resultados das propriedades física e mecânicas

\begin{tabular}{ccccccc}
\hline & \multicolumn{2}{c}{$\mathbf{f}_{\mathbf{c 0}}(\mathbf{M P a})$} & \multicolumn{2}{c}{$\mathbf{f}_{\mathbf{v} 0}(\mathbf{M P a})$} & \multicolumn{2}{c}{$\boldsymbol{\rho}_{\mathbf{a p}}\left(\mathbf{k g} / \mathbf{m}^{\mathbf{3}}\right)$} \\
$\mathrm{CP}$ & Det. & Non Det. & Det. & Non Det. & Det. & Non Det. \\
\hline 1 & 48,5 & 58,0 & 15,5 & 21,0 & 873 & 888 \\
2 & 31,4 & 35,4 & 17,4 & 19,0 & 762 & 764 \\
3 & 36,6 & 40,2 & 13,8 & 14,4 & 712 & 702 \\
4 & 39,7 & 43,5 & 11,5 & 14,2 & 907 & 944 \\
5 & 35,7 & 44,3 & 15,2 & 13,7 & 826 & 819 \\
6 & 39,4 & 50,0 & 13,7 & 13,2 & 814 & 805 \\
\hline $\mathrm{X}$ & 38,5 & 45,3 & 14,5 & 15,9 & 816 & 820 \\
$\mathrm{Sd}$ & 5,7 & 7,9 & 2,0 & 3,2 & 71 & 86 \\
$\mathrm{Cv}(\%)$ & 15 & 17 & 14 & 20 & 9 & 11 \\
\hline
\end{tabular}

Figura 10 - Resultados do teste de normalidade para as propriedades física e mecânicas investigadas
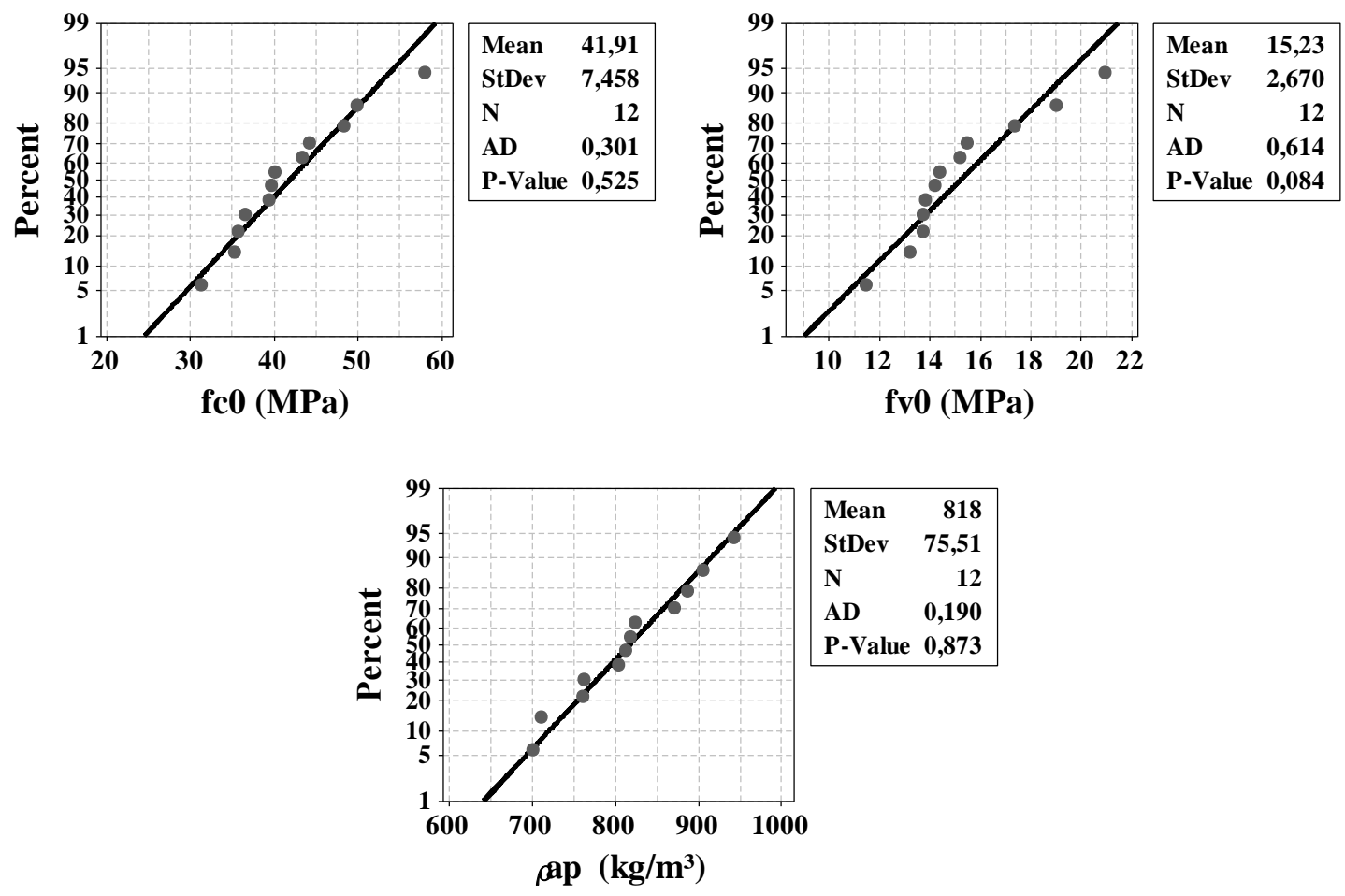

82 Andrade Junior, J. R.; Almeida, D. H. de; Almeida, T. H. de; Christoforo, A. L.; Stamato, G. C.; Lahr, R. A. R. 
Figura 11 - Resultados do teste de homogeneidade entre variâncias para a densidade aparente e para as propriedades mecânicas investigadas
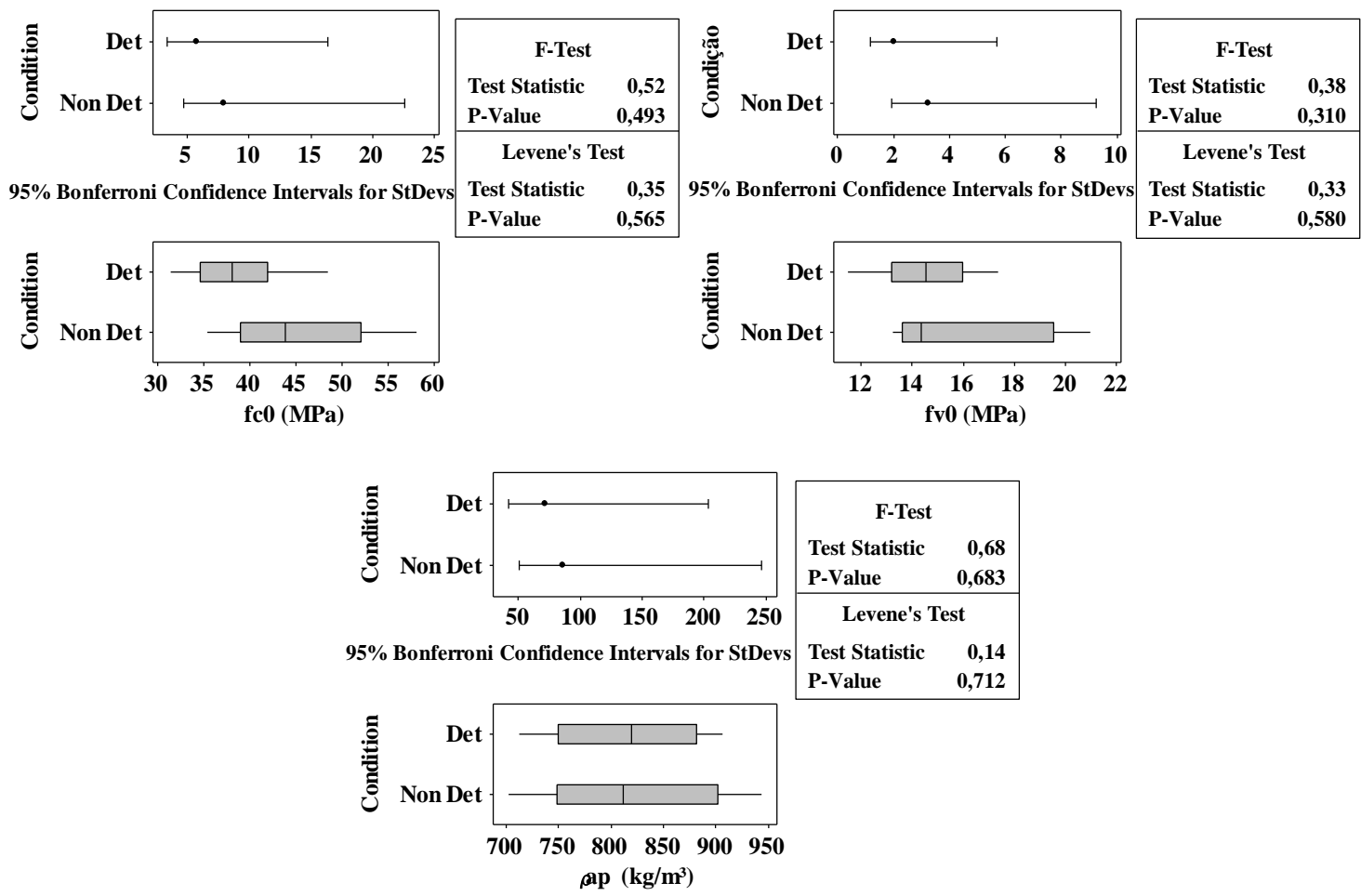

Tabela 2 - Resultados da ANOVA para a densidade aparente

\begin{tabular}{cccccc}
\hline FV & GL & SQ & MQ & F & p-valor \\
\hline Condição & 1 & 65 & 65 & 0,01 & 0,921 \\
Erro & 10 & 62651 & 6265 & & \\
Total & 11 & 62716 & & & \\
\hline
\end{tabular}

Tabela 3 - Resultados da ANOVA para a resistência na compressão paralela às fibras

\begin{tabular}{cccccc}
\hline FV & GL & SQ & MQ & F & p-valor \\
\hline Condição & 1 & 134,8 & 134,8 & 2,8 & 0,124 \\
Erro & 10 & 447,0 & 47,7 & & \\
Total & 11 & 611,8 & & & \\
\hline
\end{tabular}

Tabela 4 - Resultados da ANOVA para a resistência ao cisalhamento na direção paralela às fibras

\begin{tabular}{cccccc}
\hline FV & GL & SQ & MQ & F & p-valor \\
\hline Condição & 1 & 6,07 & 6,07 & 0,84 & 0,381 \\
Erro & 10 & 72,35 & 7,23 & & \\
Total & 11 & 78,42 & & & \\
\hline
\end{tabular}

As Tabelas 2, 3 e 4 apresentam os resultados da ANOVA para a densidade aparente, resistência na compressão paralela às fibras e resistência ao cisalhamento paralelo às fibras respectivamente. Nas tabelas têm-se GL (graus de liberdade), SQ (soma dos quadrados dos resíduos), MQ (média dos quadrados dos resíduos) e F (estatística de Snedecor).

Como osp-valores encontrados nas Tabelas 2, 3 e 4 são superiores a 0,05 , constata-se que os danos causados pelo cobrimento das madeiras pelos produtos químicos não foram significativospara nenhuma das variáveis-resposta avaliadas. 


\section{Conclusões}

Conclui-se pela análise visual das estruturas de cobertura que não existe deterioração da madeira por agentes biológicos, como cupins ou brocas. Verificou-se que, apesar de existirem algumas deteriorações superficiais das peças de madeira avaliadas, não foram significativas nas propriedades física e mecânicas investigadas.

As situações críticas encontradas na estrutura se referem à falta de estabilidade lateral do banzo superior das treliças na região dos apoios, certamente causadas pela retirada de peças de madeira do travamento que provocou a perda da estabilidade local em decorrência do aumento do índice de esbeltez.

\section{Referências}

ALMEIDA, D. H. Análise das Resistências de Embutimento e de Compressão Para Madeiras de Pínus e Eucalipto. Itapeva, 2011. 100 f. Monografia (Graduação em Engenharia Industrial Madeireira) - Campus Experimental de Itapeva, Universidade Estadual Paulista, Itapeva, 2011.

ALMEIDA, D. H. et al. Resistência ao Impacto na Flexão de Madeiras Nativa e de Reflorestamento. Madeira: Arquitetura e Engenharia, v. 12, n. 4, p. 29-36, 2011.

ALMEIDA, D. H. et al. Caracterização Completa da Madeira da Espécies Amazônica (Schizolobium amazonicum HERB) em Peças de Dimensões Estruturais. Revista Árvore, v. 37, n. 6, p. 11751181, 2013a.

ALMEIDA, D. H. et al. Full Characterization of Strength Properties of Schizolobium amazonicum Wood For Timber Structures. International Journal of Engineering \& Technology, v. 13, n. 6, p. 97-100, 2013b.

\section{ASSOCIAÇÃO BRASILEIRA DE NORMAS}

TÉCNICAS. NBR 7190: projetos de estruturas de madeiras. Rio de Janeiro, 1997.

BERTOLINI, M. S. et al. Accelerated Artificial Aging of Particleboards FromResidues of CCB Treated Pinus sp. and Castor Oil Resin. Materials Research, v. 16, n. 2, p. 293-303, 2013.
CALIL JUNIOR, C.; LAHR, F. A. R.; DIAS, A. A. Dimensionamento de Elementos Estruturais de Madeira. Barueri: Manole, 2003.

CALIL JUNIOR, C.; MOLINA, J. C. Coberturas em Estruturas de Madeira: exemplos de cálculo. São Paulo: Pini, 2010.

FERRO, F. S. et al.Verification of Test Conditions to Determine the Compression Modulus of Elasticity of Wood. International Journal of Agriculture and Forestry, v. 3, n. 2, p. 66-70, 2013.

GALVÃO, A. P. M.; JANKOWSKY, I. P. SecagemRacional da Madeira.São Paulo: Nobel, 1985.

ICIMOTO, F. H. et al. Influence of the Wood Specimen Position on Calculus of the Bending Modulus of Elasticity.International Journal of Materials Engineering, v. 3, n. 3, p. 41-46, 2013a.

ICIMOTO, F. H. et al.Physical and Mechanical Properties of ParicáWoodSpecies Treated With CCB Preservative. International Journal of Materials Engineering, v. 3, n. 4, p. 82-86, 2013b.

INSTITUTO DE PESQUISAS

TECNOLOGICAS. IPT: informações sobre madeiras. Disponível em:

<http://www.ipt.br/informacoes_madeiras3.php?m adeira $=25>$. Acesso em: 11 set. 2013.

LOGSDON, N. B.; CALIL JUNIOR, C. Influência da Umidade nas Propriedades de Resistência e Rigidez da Madeira. Cadernos de Engenharia de Estruturas, v. 18, p. 77-107, 2002.

OLIVEIRA, M. A. M. Ligações Com Pinos Metálicos em Estruturas de Madeira. São Carlos, 2001. 137f. Dissertação (Mestrado em Engenharia de Estruturas) - Escola de Engenharia de São Carlos, Universidade de São Paulo, São Carlos, 2001.

SCALIANTE, R. M. et al. Ensaio de Fluência e de Compressão Paralela às Fibras Para Situações da Madeira à Umidade de Equilíbrio e Saturada. Madeira: Arquitetura e Engenharia, v. 12, n. 3, p. 19-30, 2011. 


\section{Jairo Ribas Andrade Junior}

Escola de Engenharia de São Carlos | Universidade de São Paulo | Av. Trabalhador Sãocarlense, 400, Centro | São Carlos - SP - Brasil | CEP 13566-590 | Tel.: (016) 3373-8206 | E-mail.: jairoribas@usp.br

\section{Diego Henrique de Almeida}

Pontifícia Universidade Católica de Minas Gerais | Departamento de Engenharia Civil | Av. Padre Francis Cletus Cox, 1661, Cowtry Club | Poços de Caldas - MG- Brasil | CEP 37701-355 | Tel.: (35) 3729-9200 | E-mail.: almeida@pucpcaldas.com.br

\section{Tiago Hendrigo de Almeida}

Escola de Engenharia de São Carlos | Universidade de São Paulo | Av. Trabalhador Sãocarlense, 400, Centro | São Carlos - SP - Brasil | CEP 13566-590 | Tel.: (016) 3373-8206 | E-mail.: tiago.hendrigo@sc.usp.br

\section{André Luis Christoforo}

Departamento de Engenharia Civil | Universidade Federal de São Carlos | Rodovia Washington Luís, km 235, SP-310 | São Carlos - SP Brasil | CEP 36307-352 | Tel.: (16) 98259-6211 | E-mail.: alchristoforo@yahoo.com.br

\section{Guilherme Corrêa Stamato}

Stamade - Projeto e Consultoria em Madeira Ltda. | Rua Major José Inácio, 2920, Centro | São Carlos - SP - Brasil | CEP 13560-161 | Tel.: (16) 3116-2675 | E-mail: stamade@terra.com.br

\section{Francisco Antonio Rocco Lahr}

Escola de Engenharia de São Carlos | Universidade de São Paulo | Av. Trabalhador Sãocarlense, 400, Centro | São Carlos - SP - Brasil | CEP 13566-590 | Tel.: (016) 3373-8206 | E-mail.: frocco@sc.usp.br

\section{Revista Ambiente Construído}

Associação Nacional de Tecnologia do Ambiente Construído

Av. Osvaldo Aranha, $99-3^{\circ}$ andar, Centro

Porto Alegre - RS - Brasil

$$
\text { CEP 90035-190 }
$$

Telefone: +55 (51) 3308-4084

Fax: +55 (51) 3308-4054

www.seer.ufrgs.br/ambienteconstruido

E-mail: ambienteconstruido@ufrgs.br 